\title{
A rare case of acute transient thyroid swelling without hematoma after fine needle aspiration
}

\author{
Young Ki Kim, In Sun Goak, Yu Ji Kim, Heung Yong Jin, and Kyung Ae Lee
}

Division of Endocrinology and Metabolism, Department of Internal Medicine, Research Institute of Clinical Medicine of Jeonbuk National University-Biomedical Research Institute of Jeonbuk National University Hospital, Jeonju, Korea
A 65-year-old woman was referred to our hospital for left anterior neck swelling. UItrasound (US) revealed a normal-sized, homogenous thyroid gland with a well-defined cystic nodule $(1.91 \times 1.82 \mathrm{~cm})$ in the left lobe (Fig. 1A). US guided fine needle aspiration (FNA) of the cystic nodule was performed. No evidence of bleeding or edema was observed at the FNA site. However, about 1 hour after returning home, the patient returned to the hospital with neck pain, swelling, and shortness

Received : November 3, 2021

Revised : November 9, 2021

Accepted: November 14, 2021

Correspondence to

Kyung Ae Lee, M.D.

Tel: +82-63-250-2749

Fax: +82-63-250-2747

E-mail: kaleey@jbnu.ac.kr https://orcid.org/0000-00033700-8279

Figure 1. Serial thyroid ultrasonographic images of the patient. (A) Initial thyroid ultrasound (US) showed a normal-sized, homogeneous right thyroid lobe and a well-defined cystic nodule $(1.91 \times$ $1.82 \mathrm{~cm}$ ) in the left lobe. (B) Immediately after re-visit, US revealed a striking increase in the whole thyroid gland with heterogeneous echotexture ("cracking appearance") and the anteroposterior diameter of the isthmus increased nearly threefold compared to initial presentation. (C) Twenty-four hours following fine needle aspiration, the thyroid gland reduced to a size similar to the original size, and some swelling remained.
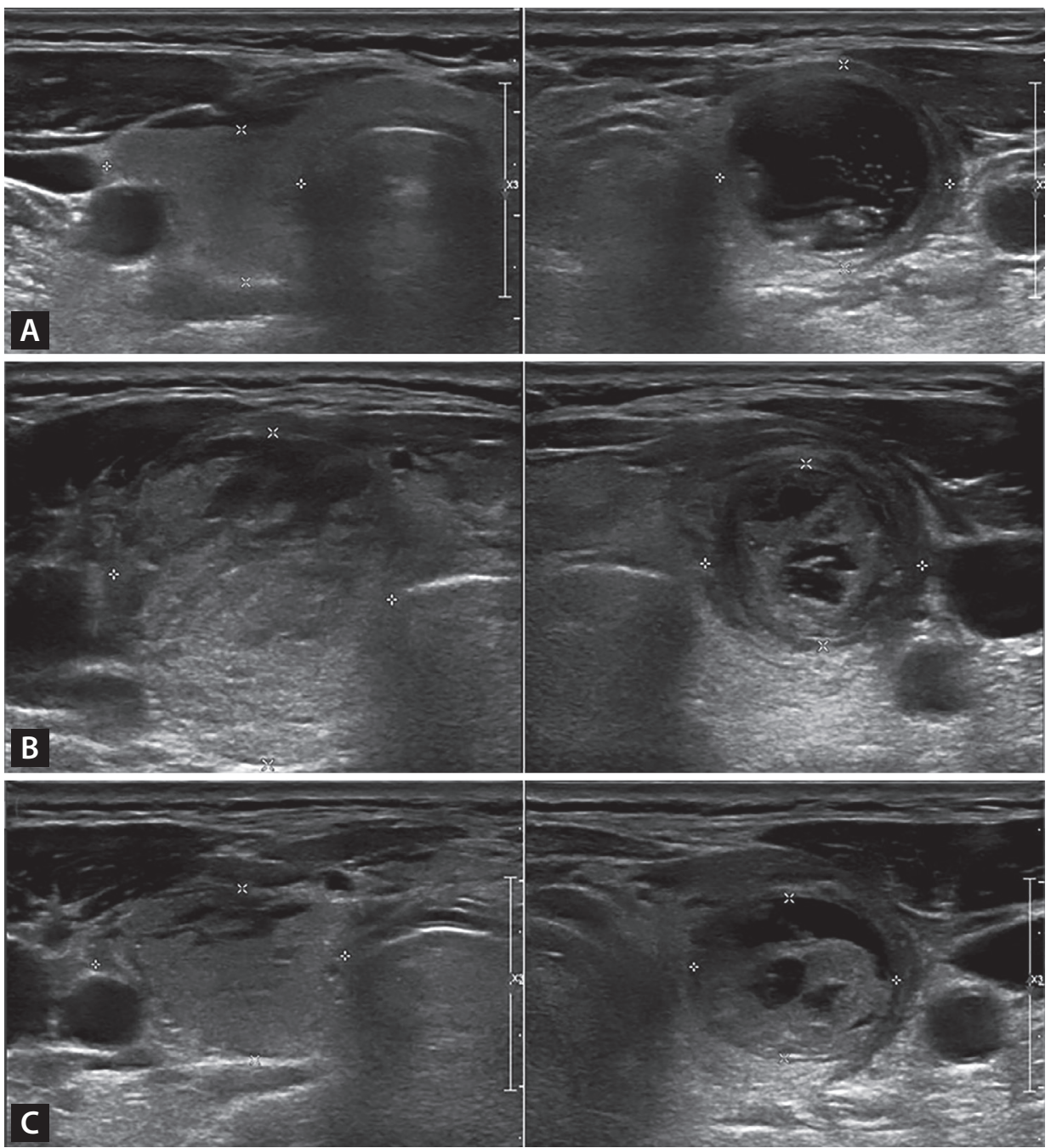

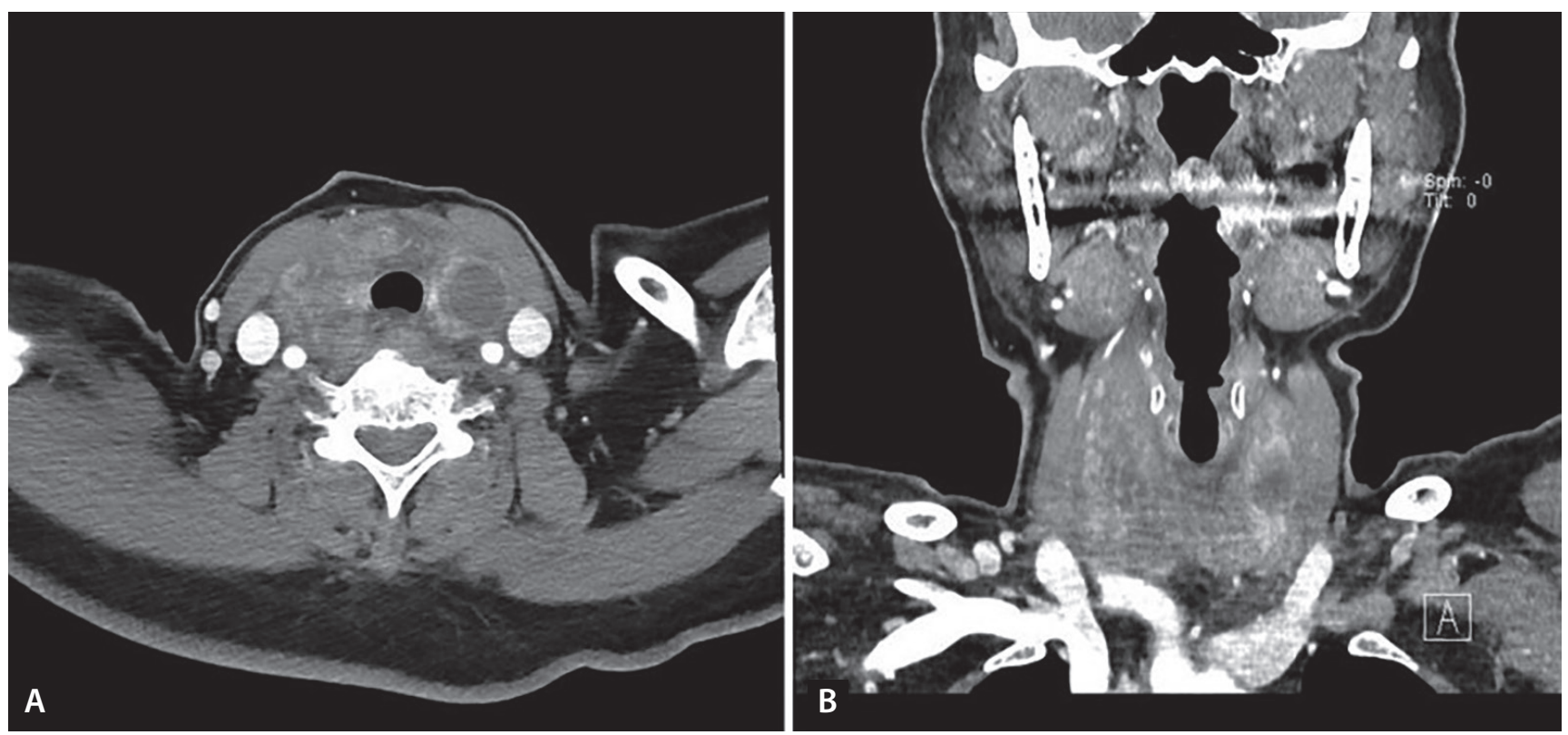

Figure 2. Contrast enhanced neck computed tomography revealed acute diffuse thyroid gland swelling with heterogenous enhancement without hematoma and airway obstruction. (A) Axial view. (B) Coronal view.

of breath. Intrathyroidal hemorrhage was suspected, and the patient was taken to the emergency room. Repeat US revealed diffuse swelling of the thyroid gland resulting in a striking increase in size (Fig. 1B). The anteroposterior diameter of the isthmus increased nearly threefold compared to initial presentation. Computed tomography of the neck also revealed diffuse thyroid swelling with heterogeneous enhancement, with no airway obstruction or hematoma (Fig. 2). Within 24 hours, the patient's symptoms improved with supportive treatment, including a single intravenous dexamethasone injection; serial follow-up US scans revealed rapid decrease in the thyroid swelling (Fig. 1C).

FNA is an invasive method widely used in the evaluation of thyroid nodules because it is simple, reliable, and safe. Post-FNA local pain or discomfort and minor hematomas are the most common complications. A few cases of acute thyroid swelling without hematoma after FNA have been reported abroad; however, no case has been reported from Korea until now. The mechanism of acute and transient thyroid swelling remains unclear. Possible explanations include intrathyroidal edema induced by endogenous substances or an allergic reaction to the needle or skin disinfectant. Despite the uncertainty of the pathophysiology and the rarity of the condition, it is important for clinicians to recognize this complication. Acute, transient thyroid swelling is generally self-limiting; therefore, awareness of this rare complication helps avoid unnecessary intervention.

\section{Conflict of interest}

No potential conflict of interest relevant to this article was reported. 